\title{
High Redshift Radio Galaxies: Laboratories for Massive Galaxy and Cluster Formation in the early Universe
}

G. Miley (Leiden), C. Carilli ${ }^{1}$ (NRAO), G.B. Taylor (UNM), C. de Breuck (ESO), \& A. Cohen (NRL)
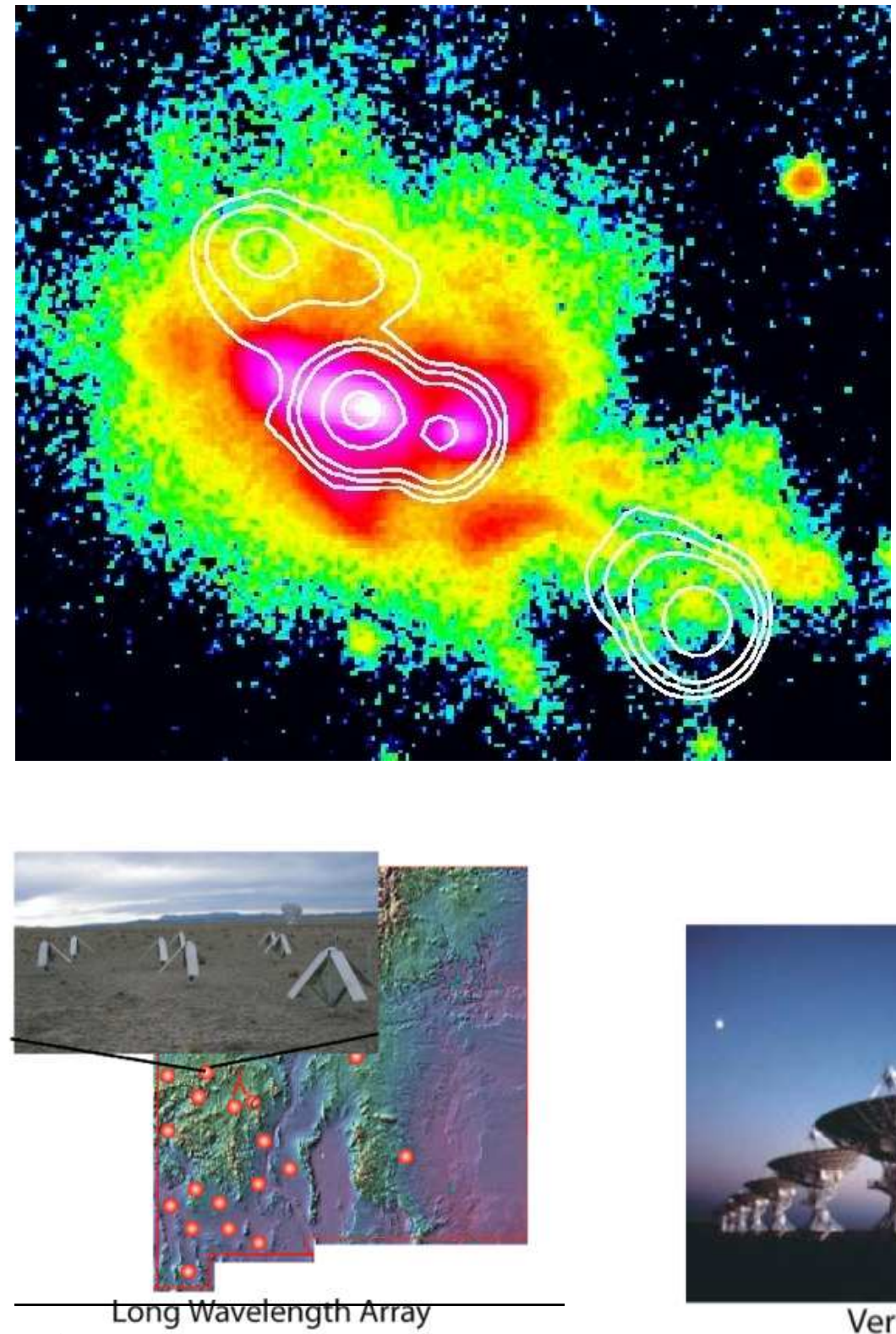

${ }^{1}$ Contact author: Chris Carilli, National Rad ccarilli@nrao.edu
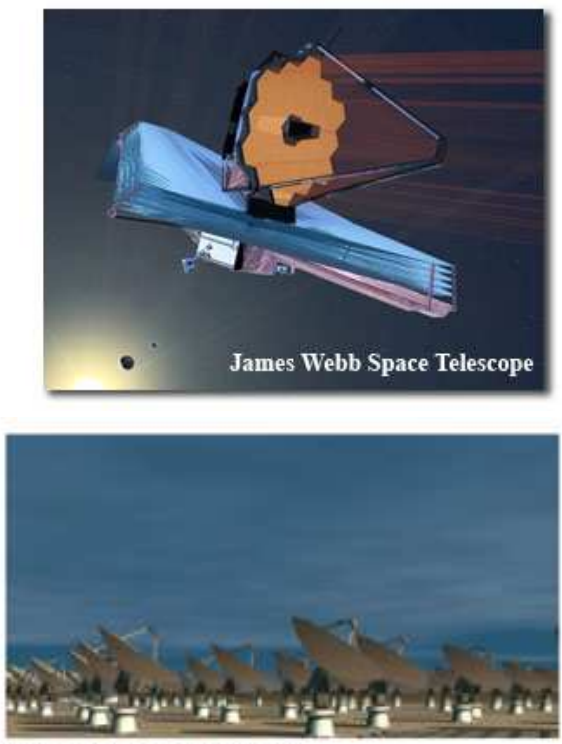

Square Kilometre Array

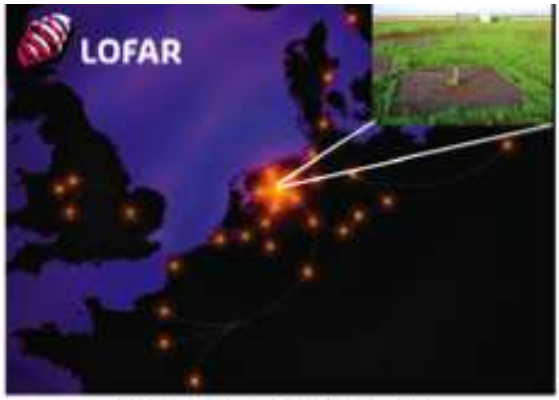

Low Frequency Array

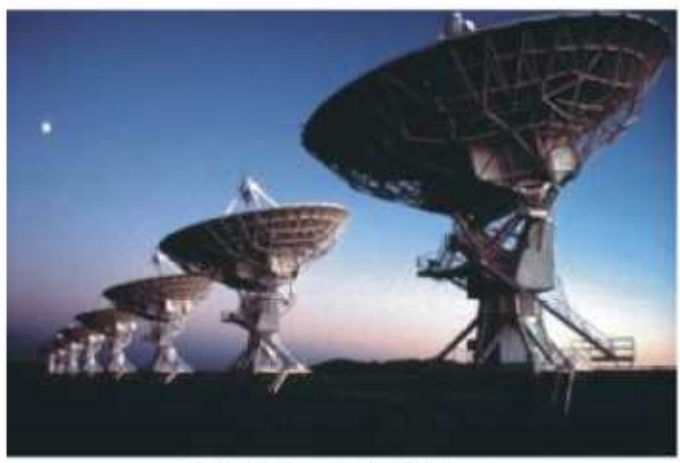

Very Large Array

, Socorro, NM, 87801, 


\section{Beacons to massive galaxy and cluster formation in the early Universe}

High redshift radio galaxies (HzRGs, $\mathrm{z}>2$ ) are among the largest, most luminous, most massive, and most beautiful objects in the Universe. They are generally identified from their radio emission, thought to be powered by accretion of matter onto supermassive black holes in the nuclei of their host galaxies. Further observations show that they are energetic sources of radiation throughout most of the electromagnetic spectrum. Figure 1 shows the spectral energy distribution (SED) of a typical HzRG from radio to X-ray wavelengths, together with a decomposition into various observable HzRG constituents - relativistic plasma, gas and dust, stars and the active galactic nuclei (AGN).

In a recent review article Miley \& de Breuck (9) present an extensive analysis of the properties and implications of HzRGs, including a detailed description of the major emitting components which provide important diagnostics about various physical constituents of the early Universe. A list of known HzRG building blocks is given in Table 1, together with a summary of techniques used to study them. Also included are a list of the resultant diagnostics, some useful references (see Miley \& De Breuck), and our best estimate for the typical mass of the component in HzRGs. As can be seen in Table 1, several constituents of HzRGs are inferred to be extremely massive, including old stars (up to $\sim 10^{12} \mathrm{M}_{\odot}$ ), hot gas (up to $\sim 10^{12} \mathrm{M}_{\odot}$ ) and molecular gas (up to $\sim 10^{11} \mathrm{M}_{\odot}$ ).

Because they are highly luminous and (unlike quasars) spatially resolvable from the ground, most components of HzRGs provide important diagnostic information about the spatial distributions of processes within HzRGs and their environment. The fact that the different constituents are present in the same objects and that the interrelationships and interactions between them can be studied make distant radio galaxies unique laboratories for probing massive galaxy and cluster formation in the early Universe.

\section{The Spiderweb Galaxy - a case study}

The Spiderweb Galaxy (MRC 1138-262), at a redshift of $z=2.2$, is one of the most intensively studied HzRGs. This object provides a useful case study for illuminating several important physical processes that may occur generally in the evolution of the most massive galaxies. Because the Spiderweb Galaxy is (i) relatively close-by, (ii) one of the brightest known HzRGs and (iii) the HzRG with the deepest HST optical image, it is an important laboratory for testing simulations of forming massive galaxies at the centers of galaxy clusters.

This large galaxy has several of the properties expected for the progenitor of a dominant cluster galaxy. The IR luminosity corresponds to a stellar mass of $\sim 10^{12} \mathrm{M}_{\odot}$, implying that MRC 1138-262 is one of the most massive galaxies known at $\mathrm{z}>2$. The host galaxy is surrounded by a giant $\operatorname{Ly} \alpha$ halo and embedded in dense hot ionized gas with an ordered magnetic field causing rotation measures up to $\sim 6000 \mathrm{rad} \mathrm{m}^{-2}$. The radio galaxy is associated with a $3 \mathrm{Mpc}$-sized structure of galaxies, of estimated mass $>2 \times 10^{14} \mathrm{M}_{\odot}$. This type 
Table 1. Constituents of distant radio galaxies

\begin{tabular}{|c|c|c|c|c|}
\hline Constituent & Observable & Typical Diagnostics & Refs. & $\begin{array}{l}\text { Mass } \\
\left(\mathrm{M}_{\odot}\right)\end{array}$ \\
\hline \multirow[t]{2}{*}{$\begin{array}{l}\text { Relativistic } \\
\text { plasma }\end{array}$} & Radio continuum & $\begin{array}{l}\text { Magnetic field, age, energetics, } \\
\text { pressure, particle acceleration. } \\
\text { Jet collimation and propagation }\end{array}$ & 1,2 & \\
\hline & X-ray continuum & $\begin{array}{l}\text { Magnetic field, equipartition, } \\
\text { pressures }\end{array}$ & $3,4,1$ & \\
\hline \multirow{2}{*}{$\begin{array}{l}\text { Hot ionized gas } \\
\mathrm{T}_{e} \sim 10^{7}-10^{8} \mathrm{~K} \\
\mathrm{n}_{e} \sim 10^{-1.5} \mathrm{~cm}^{-3}\end{array}$} & $\begin{array}{l}\text { Radio } \\
\text { (de)polarisation }\end{array}$ & Density, magnetic field, & 1 & \multirow[t]{2}{*}{$10^{11-12}$} \\
\hline & X-rays & Temperature, density mass & & \\
\hline \multirow{2}{*}{$\begin{array}{l}\text { Warm ionized gas } \\
\mathrm{T}_{e} \sim 10^{4}-10^{5} \mathrm{~K} \\
\mathrm{n}_{e} \\
10^{0.5-1.5} \mathrm{~cm}^{-3}\end{array}$} & $\begin{array}{l}\text { UV-optical } \\
\text { emission lines }\end{array}$ & $\begin{array}{l}\text { Temperature, density, kinemat- } \\
\text { ics, mass, ionisation, metallicity, } \\
\text { filling factor }\end{array}$ & $5,6,7,8$ & \multirow[t]{2}{*}{$10^{9-10.5}$} \\
\hline & $\begin{array}{l}\text { Nebular } \\
\text { continuum }\end{array}$ & SED contamination & 9,10 & \\
\hline \multirow{2}{*}{$\begin{array}{l}\text { Cool atomic gas } \\
\mathrm{T}_{s} \sim 10^{3} \mathrm{~K} \\
\mathrm{n}(\mathrm{HI}) \sim 10^{1} \mathrm{~cm}^{-3}\end{array}$} & HI absorption & $\begin{array}{l}\text { Kinematics, column densities, } \\
\text { spin temperature, sizes, mass }\end{array}$ & 11,8 & \multirow[t]{2}{*}{$10^{7-8}$} \\
\hline & $\begin{array}{l}\text { UV-optical } \\
\text { absorption lines }\end{array}$ & $\begin{array}{l}\text { Kinematics, mass, column den- } \\
\text { sities, metallicity }\end{array}$ & $\begin{array}{l}8,12 \\
13,14\end{array}$ & \\
\hline $\begin{array}{l}\text { Molecular gas } \\
\mathrm{T} \sim 50-500 \mathrm{~K} \\
\mathrm{n}\left(\mathrm{H}_{2}\right)>10^{2} \mathrm{~cm}^{-3} \\
\end{array}$ & $\begin{array}{l}\text { (Sub)millimeter } \\
\text { lines }\end{array}$ & Temperature, density, mass & 15 & $10^{10-11}$ \\
\hline \multirow[t]{2}{*}{$\begin{array}{l}\text { Dust } \\
\mathrm{T} \sim 50-500 \mathrm{~K}\end{array}$} & $\begin{array}{l}\text { UV-optical } \\
\text { polarisation }\end{array}$ & $\begin{array}{l}\text { Dust composition, scattering, } \\
\text { mass, hidden quasar }\end{array}$ & 1617 & \multirow[t]{2}{*}{$10^{8-9}$} \\
\hline & $\begin{array}{l}\text { (Sub)millimeter } \\
\text { continuum }\end{array}$ & $\begin{array}{l}\text { Temperature, mass, heating } \\
\text { source }\end{array}$ & 18 & \\
\hline $\begin{array}{l}\text { Old stars } \\
\mathrm{t}>1 \mathrm{Gyr}\end{array}$ & $\begin{array}{l}\text { Optical to near } \\
\text { IR continuum }\end{array}$ & Age, mass, formation epoch & 19 & $10^{11-12}$ \\
\hline \multirow[t]{2}{*}{$\begin{array}{l}\text { Young stars } \\
\mathrm{t}<0.5 \text { Gyr }\end{array}$} & UV-optical & Star formation rates, ages & 20,8 & \multirow[t]{2}{*}{$10^{9-10}$} \\
\hline & $\operatorname{Ly} \alpha$ & Star formation rate & 20 & \\
\hline $\begin{array}{l}\text { Quasar (hidden } \\
\text { or dormant) }\end{array}$ & $\begin{array}{l}\text { UV-optical } \\
\text { polarisation } \\
\text { broad lines }\end{array}$ & Luminosity & 21,22 & \\
\hline $\begin{array}{l}\text { Supermassive } \\
\text { black hole }\end{array}$ & $\begin{array}{l}\text { Extended radio, } \\
\text { Quasar }\end{array}$ & Formation, evolution & 23,24 & $\sim 10^{9}$ \\
\hline
\end{tabular}




\section{Restframe Wavelength ( $\mu \mathrm{m})$}

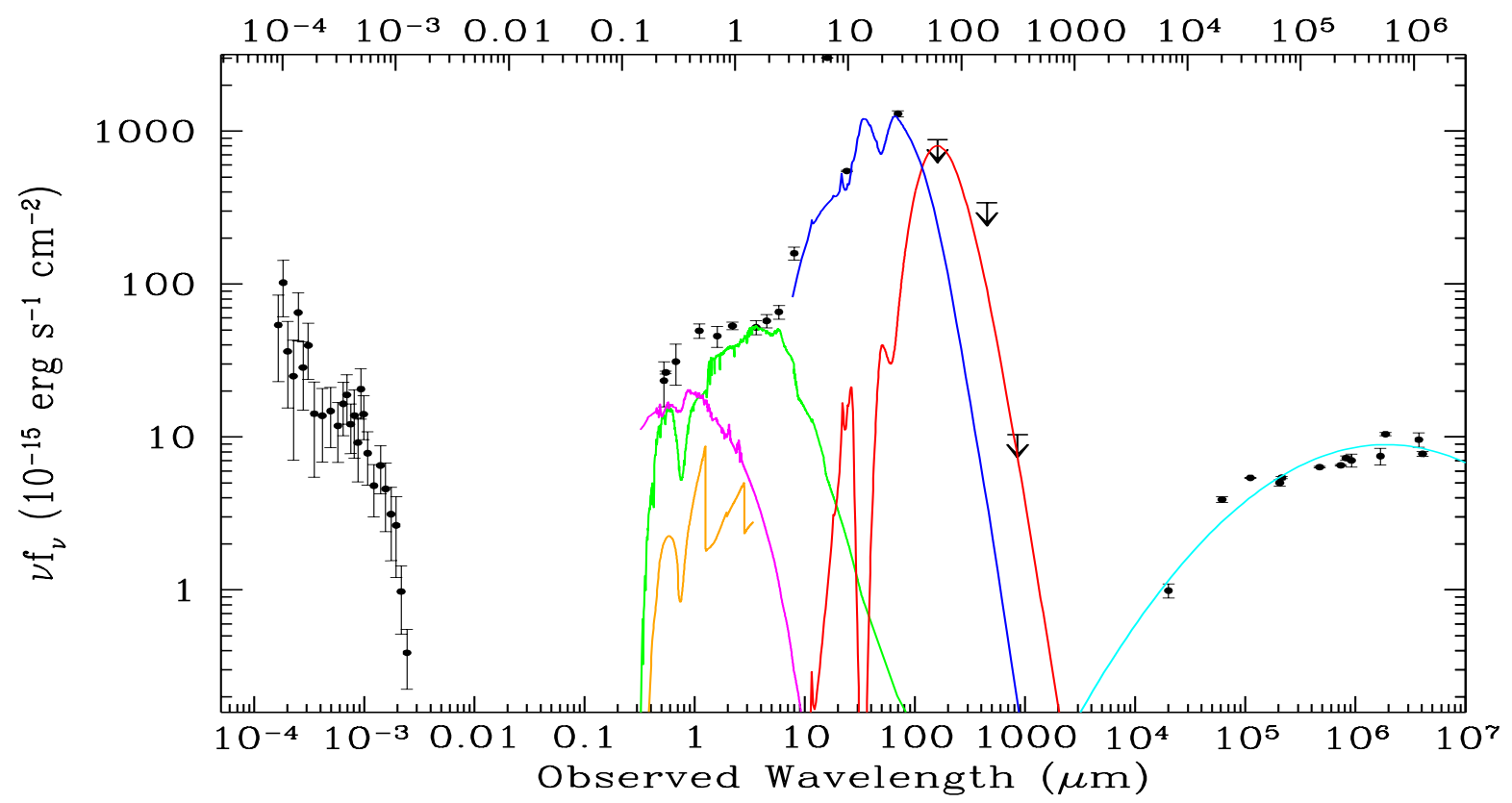

Fig. 1. - Spectral energy distribution (SED) of the continuum emission from the HzRG $4 \mathrm{C} 23.56$ at $\mathrm{z}=2.5$, illustrating the contributions from the various constituents. [From De Breuck et al. in preparation]. Colored lines show the decomposition of the SED into individual components, under many assumptions. Cyan = radio synchrotron; Black = Absorbed nonthermal X-ray AGN; Yellow = nebular continuum; Blue = AGN-heated thermal dust emission; Red $=$ Starburst-heated dust emission; Green $=$ Stars; Magenta $=$ scattered quasar. The addition of the overlapping modeled components fits the SED well.

of structure is the presumed antecedent of present-day rich clusters.

The beautiful ACS Hubble image of the Spiderweb Galaxy is shown in Figure 2. This figure illustrates the structures of the radio, warm gas and stellar components in a relatively nearby HzRG. It also provides dramatic evidence that tens of satellite galaxies were merging into a massive galaxy, 10 Gyr ago. The morphological complexity and clumpiness agrees qualitatively with predictions of hierarchical galaxy formation models, and illustrates this process in unprecedented detail. Ly $\alpha$ spectroscopy shows relative velocities of several hundred $\mathrm{km} \mathrm{s}^{-1}$, implying that the satellite galaxies ("flies") will traverse the $100 \mathrm{kpc}$ extent of the Spiderweb many times in the interval between $\mathrm{z} \sim 2.2$ and $\mathrm{z} \sim 0$, consistent with the merger scenario.

An intriguing aspect of the Spiderweb Galaxy is the presence of faint diffuse emission between the satellite galaxies. Approximately 50\% of the ultraviolet light from the Spiderweb Galaxy is in diffuse "intergalactic" light, extending over a $\sim 60 \mathrm{kpc}$ diameter halo. The luminosity in diffuse light implies that the emission is dominated by young stars with a star formation rate of $>80 M_{\odot} \mathrm{yr}^{-1}$. Under reasonable assumptions, the diffuse emission seen in the Spiderweb Galaxy could evolve into the CD envelopes seen in many dominant cluster galaxies at low redshifts. 

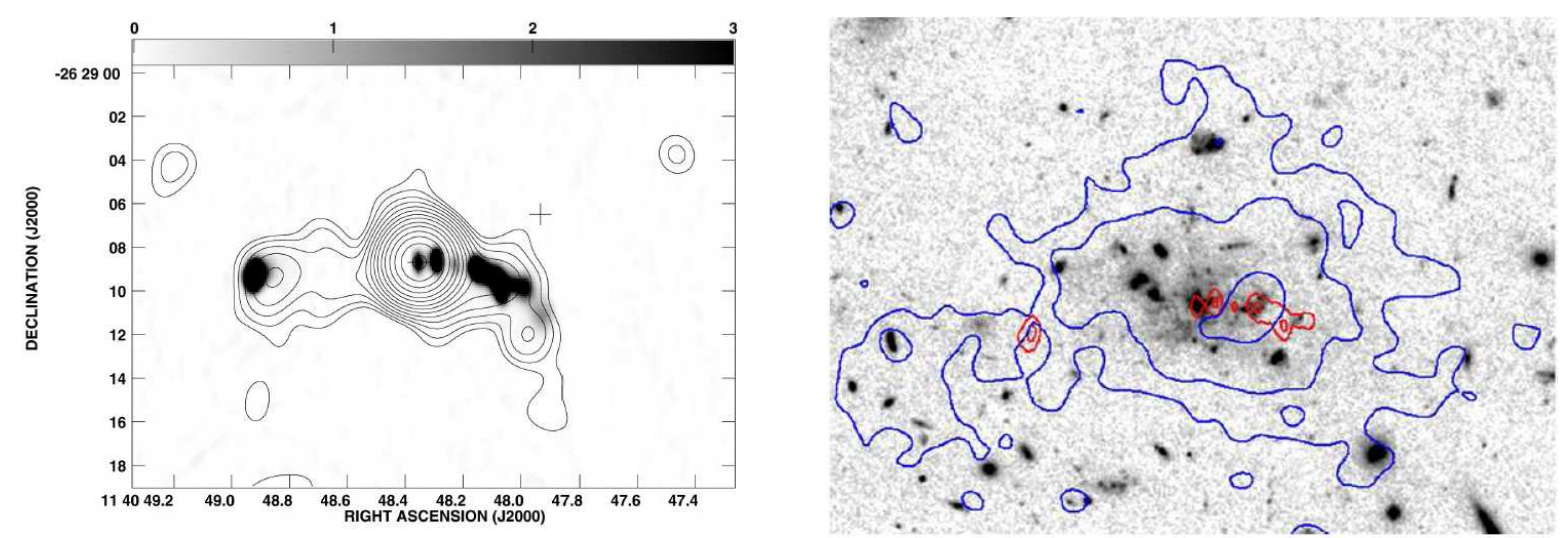

Fig. 2.- Left: X-ray emission from the Spiderweb Galaxy, PKS 1138-262 at z $=2.2$, observed with the Chandra X-ray Telescope (2). X-ray contours are superimposed on a VLA gray scale representation of the $5 \mathrm{GHz}$ radio continuum emission at 0.5 " resolution. The cross marks the position of the radio galaxy nucleus. Note that the X-ray and radio emission are aligned with each other. Right: The Spiderweb Galaxy. Deep Hubble image of the core of the MRC 1138-262 protocluster at $\mathrm{z}=2.2$ obtained with the Advanced Camera for Surveys. [From (8). Superimposed on the HST image are contours of Ly $\alpha$ (blue, resolution 1") obtained with ESO's very Large Telescope (VLT), delineating the gaseous nebula and radio $8 \mathrm{GHz}$ contours (red, resolution 0.3 ") obtained with NRAO's VLA, delineating the non-thermal radio emission. The gaseous nebula extends for $>200 \mathrm{kpc}$ and is comparable in size with the envelopes of $\mathrm{cD}$ galaxies in the local Universe.

The total mass of all the flies in the Spiderweb, derived from their UV luminosities (assuming 1Gyr starbursts), is less than a tenth of the mass of the whole galaxy obtained from its IR luminosity. Because the UV emission is produced by ongoing star formation and the IR emission by old stars, this implies that most of the galaxy mass may already have been assembled by $\mathrm{z} \sim 2.2$, consistent with downsizing scenarios.

Merging, downsizing and feedback are all likely to be occurring simultaneously in the Spiderweb Galaxy. Merging is a plausible fueling source for the nuclear supermassive black holes that produce the radio sources. Pressure from these radio sources is sufficient to expel a large fraction of gas from the galaxies, thereby quenching star formation. Because radio lifetimes are relatively short (few $\times 10^{7} \mathrm{yr}$ ), all massive ellipticals may have gone through a similar short but crucial radio-loud phase during their evolution.

An unexpected feature of the HST image is that there is a significant excess of faint satellite galaxies with linear structures (8). These galaxies (linear "flies") have similar morphologies (e.g. chains and tadpoles) to the linear galaxies that dominate resolved faint galaxies $\left(\mathrm{i}_{775}>24\right)$ in the Hubble Ultra Deep Field (UDF) $(4,10)$. Although linear galaxies must be an important constituent of the earliest galaxy population, their nature is poorly understood. Their presence in a merging system is relevant for theories of their formation. 
In the Spiderweb Galaxy the motions of the flies with velocities of several hundred $\mathrm{km} \mathrm{s}^{-1}$ through the dense gaseous halo, perturbed by superwinds from the nucleus $(1,13)$ and the radio jet, and/or more frequent interaction of the flies with each other would result in shocks. The shocks would then lead to Jeans-unstable clouds, enhanced star formation along the direction of motion and to chain and tadpole morphologies $(11,8)$.

\section{Unanswered questions}

There are still many aspects of HzRGs that are not understood, including:

- What is the particle acceleration mechanism in the relativistic plasma and why do HzRGs have much steeper radio spectra than nearby radio sourcesi

- What caused the most luminous radio galaxies and quasars to become virtually extinct between $\mathrm{z} \sim 2$ and the present?

- What is the nature, extent and kinematics of the hot gas, one of the most massive and least studied constituents of HzRGs?

- What is the origin of the Ly $\alpha$ halo that appears to be falling into the HzRG and how is this warm gas (filling factor $\sim 10^{-5}$ ) distributed with respect to the hotter and colder gas (filling factors $\sim 1$ )?

- What are the processes by which the radio jets interact with the gas and trigger starburst, and how important is jet-induced star formation in the early Universe?

- What are the temperature and densities and composition of the molecular gas and dust? What are their spatial distributions and what does this imply for the star formation histories?

- What is the relative importance of AGN and starburst heating in the mid-IR emission?

- What effect does galaxy merging have on star formation and what physical effects are responsible for downsizing?

- What effect does feedback between the AGN and the galaxy have on the evolution of HzRGs and the general evolution of massive galaxies?

- What is the detailed mechanism by which the SMBHs produce quasars and jets?

- How and why do the AGN/quasars vary on long time-scales?

- How is the SMBH built up and what role does merging play in this evolution?

- What is the size distribution of radio-selected protoclusters and what is is the topology of the cosmic web in the neighbourhood of the HzRGs?

- How do the various populations and constituents of distant protoclusters evolve and eventually become virialised clusters?

- What is the radio luminosity function of galaxies in radio-selected protoclusters? Is the radio emission from the protocluster galaxies an important contributor to forming the radio halos, seen at the centres of many nearby rich clusters? What is the strength and configuration of the protocluster magnetic fields? 


\section{A promising future}

There are good prospects for making progress on the many unknown aspects of HzRGs during the next decade. Since its inception, the study of radio galaxies has been observationally driven. Several forefront astronomical facilities are now being constructed or planned that will give new insights into the nature of HzRGs and their environments.

First, with a combination of sensitivity and spatial resolution, the new low-frequency radio arrays, LOFAR and the LWA, will open up the frequency window below $\sim 50 \mathrm{MHz}$ for HzRG studies. The LWA will survey the sky to unprecedented depth at low-frequencies and will therefore be sensitive to the relatively rare radio sources that have extremely steep spectra. Because of the $\alpha$ vs z relation, the LWA is likely to detect HzRGs at $\mathrm{z} \sim 8$, if they exist. Studies of detailed low-frequency spectra and their spatial variations will provide new information about the mechanism responsible for the $\alpha$ vs z relation. Presently combinations of the new radio surveys with planned new deep optical and infrared wide-field surveys, such as PAN-STARRS (6) and those with the VST and VISTA will be used to identify HzRGs and provide photometric data.

Another task for sensitive radio arrays, such as LWA, LOFAR, the EVLA and eventually the Square Kilometre Array (SKA), will be to survey the radio emission of galaxies in protoclusters. The new arrays will study radio emission produced by relativistic jets and be able to detect and investigate radio emission from the brightest star forming galaxies.

Secondly, ALMA and the EVLA, with their unprecedented sensitivities and resolutions at millimetre and sub-millimetre wavelengths, will revolutionize the study of molecular gas and dust. Several different CO transitions can be observed, allowing entire "CO ladders" to be constructed and the density and temperature structure of the molecular gas to be unraveled. Fainter molecular lines can be used to trace even denser gas than that studied until now. Important information about the dust composition and the gas to dust ratios is likely to be obtained.

ALMA's sensitivity at millimetre wavelengths should also facilitate observations of the atomic CI Carbon lines in HzRGs. This would provide an important constraint on the global metalicity of the gas. The fine-structure line of $\mathrm{C}^{+}$at $\lambda_{\text {rest }}=157.74 \mu \mathrm{m}$ line is one of the main cooling lines in nearby galaxies, and has now also been detected in several of the most distant quasars known (12).

Thirdly, we can expect considerable progress in disentangling the detailed evolutionary history both of HzRGs and of radio-selected protoclusters. This evolutionary detective work will be pursued by combining spectroscopic data from the next generation of spectrographs on 8m-class telescopes with imaging results from the new camera, WFC3 (7), on the Hubble Space Telescope. For example, the detection of supernovae in $\mathrm{z} \sim 2$ protoclusters will become possible. On a longer timescale, tracing the detailed history of the formation and evolution of HzRGs and the surrounding protoclusters will be helped enormously with the advent of 
$30 \mathrm{~m}$-class ground-based telescopes in the optical and near-infrared and the James Webb Space Telescope (JWST) in the near and mid-infrared.

Fourthly, the next generation X-ray telescope, such as XEUS or Constellation-X, will make observations of X-rays from HzRGs an important tool for studying galaxy formation. It will have sufficient sensitivity to perform spectroscopic studies of hot gas in HzRGs. The 0.3 - $10 \mathrm{keV}$ X-ray band contains the inner (K-shell) lines for all of the abundant metals from carbon to zinc as well as many L-shell lines. These atomic transitions provide important new plasma diagnostics of the HzRG hot gas.

Fifthly, and perhaps most exciting, the potential discovery of HzRGs with z $>6$ could open up a unique new window for studying the very early Universe during the "Epoch of Reionisation". Recent observational constraints suggest that cosmic reionization may have taken place between $\mathrm{z} \sim 11$ and $\mathrm{z} \sim 6$. The existence of HzRGs within the near edge of cosmic reionization could be used as sensitive probes of intermediate- to small-scale structures in the neutral IGM, through redshifted HI absorption observations $(3,5)$, complementary to the very large scale that can be studied in HI emission.

\section{REFERENCES}

1 Armus, L., Heckman, T. M., \& Miley, G. K. 1990, ApJ, 364, 471

2 Carilli, C. L. et al. 2002, ApJ, 567, 781

3 Carilli et al. 2002, ApJ, 577, 22

4 Elmegreen, D. M. et al. 2005, ApJ, 631, 85

5 Furlanetto, S. 2006, MNRAS, 371, 867

6 Hodapp, K. W., et al. 2004, Astronomische Nachrichten, 325, 636

7 Leckrone, D. S. 1998, BAAS, 30, 861

8 Miley, G. K., et al. 2006, ApJ, 650, L29

9 Miley, G.K., \& De Breuck, C. 2008, A\&A Rev., 15, 67

10 Straughn, A. N. et al. 2006, ApJ, 639, 724

11 Taniguchi, Y., \& Shioya, Y. 2001, ApJ, 547, 146

12 Walter, F. et al. 2009, Nature, 475, 699

13 Zirm, A. W., et al. 2005, ApJ, 630, 68

This preprint was prepared with the AAS IATEX macros v5.2. 